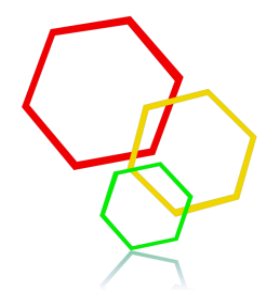

Вісник Дніпропетровського університету. Серія Хімія

Bulletin of Dnipropetrovsk University. Series Chemistry

p-ISSN 2306-871X, e-ISSN 2313-4984

journal homepage: http://chemistry.dnu.dp.ua

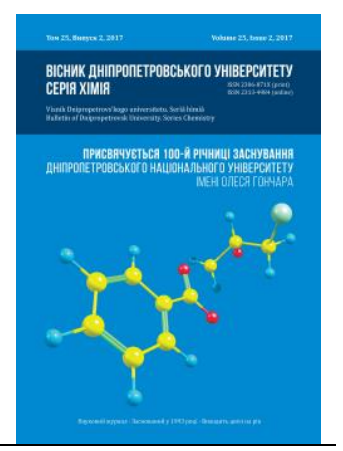

UDC 543.612.2+543.422.7

\title{
SPECTROPHOTOMETRIC DETERMINATION OF SCANDIUM IN ALLOY 01570
}

\author{
Anna Yu. Chernyavskaya, Larisa P. Sidorova, Lidiya A. Ivanitsa*, Tatyana S. Chmilenko, \\ Fedor A. Chmilenko
}

Oles Honchar Dnipro National University, Ukraine, Dnipro, 72 Gagarina Avenue, 49010

Received 17 November 2017; revised 23 November 2017; accepted 21 December 2017

\begin{abstract}
The complexation of scandium with chromazurol $S$ and eriochromcyanine $R$ in the presence of cationic polyacrylamide (brand «FO»)with different charge density was investigated by spectrophotometry. The formation of ternary metal-polymeric complexes of organic reagent - polyacrylamide - scandium was established. The composition of ternary metal-polymer complexes determined by the ternary Gibbs-Rosenbaum diagram were 6:3:1 for ECC-F04650-Sc, 1:1:1 and 1:3:1 for XAS-F04400-Sc. The system of ECC-F04700-Sc is proposed as analytical form for spectrophotometric determination of scandium due to high molar absorbtivity for this complex determined from the slope of the calibration graph $\left(7.2 \cdot 10^{4} \mathrm{~mol}^{-1} \cdot \mathrm{L}^{\cdot} \mathrm{cm}^{-1}\right)$. The proposed technique allows to determine $\mathrm{Sc}^{3+}$ in the range of concentrations $9-90 \mu \mathrm{g} / \mathrm{L}$. The limit of determination is $5.5 \mu \mathrm{g} / \mathrm{L}$. The sample of scandium-containing alloy 01570 was analyzed by proposed technique $\left(S_{r}=0.054\right)$. The scandium was preliminarily separated from disturbing components by the precipitation with $\mathrm{NaOH}$. The correctness of the rezults is confirmed by the standard procedure technique.
\end{abstract}

Key words: organic reagent; polyelectrolyte; scandium; ternary metal-polymer complex; polyacrylamide flocculant

\section{СПЕКТРОФОТОМЕТРИЧНЕ ВИЗНАЧЕННЯ СКАНДІЮ В СПЛАВІ 01570}

\author{
Анна Ю. Чернявська, Лариса П. Сидорова, Лідія О. Іваниця, Тетяна С. Чмиленко, \\ Федір 0. Чмиленко \\ Дніпровський національний університет імені Олеся Гончара, Украӥна, м Дніпро, пр. Гагаріна, 72, 49010
}

\section{Анотація}

В роботі розглянуто комплексоутворення скандію з хромазуролом $S$ та еріохромціаніном $R$ в присутності катіонних поліакриламідів марки FO 3 різною густиною заряду. Встановлено утворення потрійних металополімерних комплексів органічний реагент - поліелектроліт - скандій. Визначено склад вище зазначенних потрійних металополімерних комплексів. Запропоновано методику спектрофотометричного визначення скандію після попередньо виділення осадженням $3 \mathrm{NaOH}$ у вигляді аналітичної форми EXЦ - FO 4700 - Sc у інтервалі концентрацій Sc ${ }^{3+} 9$ - 90 мкг/л. Методику апробовано при аналізі сплаву 01570.

Ключові слова: органічний реагент; поліелектроліт; скандій; потрійний металополімерний комплекс; поліакриламідний флокулянт

*Corresponding author: Tel.: 380567766014; e-mail address: lidiya.ivanitsa@gmail.com

(C) 2017 Oles Honchar Dnipro National University

doi: 10.15421/081712 


\title{
СПЕКТРОФОТОМЕТРИЧЕСКОЕ ОПРЕДЕЛЕНИЕ СКАНДИЯ В СПЛАВЕ 01570
}

\author{
Анна Ю. Чернявская, Лариса П. Сидорова, Лидия А. Иваница, Татьяна С. Чмиленко, \\ Федор А. Чмиленко \\ Днепровский национальный университет имени Олеся Гончара, Украина, г. Днипро, пр. Гагарина, 72, 49010
}

\section{Аннотация}

В работе рассмотрено комплексообразование скандия c хромазуролом $S$ и эриохромцианином $R$ B присутствии катионных полиакриламидов марки FO c различной плотностью заряда. Установлено образование тройных металлополимерных комплексов органический реагент - полиэлектролит - скандий. Определен состав указанных выше тройных металлополимерных комплексов. Предложена методика спектрофотометрического определения скандия после предварительного отделения осаждением с NaOH в виде аналитической формы ЭХЦ - FO 4700-Sc в интервале концентраций Sc ${ }^{3+9}$ - 90 мкг/л. Методику апробировано при анализе сплава 01570.

Ключевые слова: органический реагент; полиэлектролит; скандий; тройной металлополимерный комплекс, полиакриламидный флокулянт

\section{Введение}

Скандий - перспективный конструкционный материал для авиа- автомобиле- и ракетостроения в силу своей высокой теплостойкости, легкости, высокой прочности и химической стойкости. Практически примесь до $0.35 \%$ скандия увеличивает теплостойкость и прочность алюминиевых сплавов, таких как АМг6 (1570), 1460, А356 до 3 раз [1-5].

Для определения скандия в промышленных объектах используют спектрофотометрический [6; 7], атомноабсорбционный [8], эмисионный спектрографический [9] анализ. Наиболее распространенным является спектрофотометрический метод [10]. Для фотометрического метода количественного определения скандия в сложных объектах были предложены различные органические реагенты, имеющие высокую чувствительность, но сравнительно низкую селективность. Среди них эриохромцианин $\mathrm{R}$ (ЭХЦ) [11; 12], хромазурол S (XAS) [13; 14], арсеназо III [15], хлорцианформазан и другие реагенты [16].

Для повышения чувствительности и селективности спектрофотометрического определения скандия применяют экстракцию различными органическими растворителями, такими как Cyanex 272 [15], бис (трифлуорометилсульфонил)имид [17], изопропиловый спирт - вода - натрия нитрат - калия роданид [18; 19]. При выборе экстрагента для скандия, наряду с такими характеристиками, как полнота извлечения и избирательность, необходимо учитывать токсичность и летучесть, а также экономичность. Этим требованиям наиболее полно отвечают двухфазные водные системы на основе водорастворимых полимеров типа полиэтиленгликолей (ПЭГ), которые используются для разделения, концентрирования и определения редких, радиоактивных элементов, например системы полиэтиленгликоль - натрия (аммония) нитрат - вода [20].

Еще одним перспективным направлением повышения чувствительности и селективности спектрофотометрических методик является применение поверхностноактивных веществ (ПАВ), таких как цетилпиридиний хлорид (ЦПХ) [11; 13] и полиэлектролитов [14; 21-22]. При введении полиэлектролитов или ПАВ растут молярные коэффициенты светопоглощения и повышается контрастность реакций. Как правило, есть две модели механизма действия ПАВ: солюбилизация и формирование тройных комплексов. В присутствии ПАВ гидрофобные молекулы становятся растворимыми в водной среде [21; 23]. Приведены данные комплексообразования скандия с ЭХЦ в присутствии катионных ПАВ: ЦПХ, цетилтриметиламмоний бромида, додецилтриметиламмоний бромида, неионного ПАВ - тритона X-100, анионного додецилсульфата натрия. С ЭХЦ, модифицированным ПАВ, скандий определяют в синтетических сплавах и водах [11], моназите [12]. Исследовано комплексообразование скандия с ХАЗ в присутствии ЦПХ, смеси ЦПХ и тритона X-100, смеси катионных и анионных ПАВ, неионных ПАВ. Скандий определяют с XAS, модифицированным ПАВ, в искусственных рудах, синтетических образцах оксида лантана и иттрия [11]. Описана реакция 
скандия с хромазуролом $\mathrm{S}$ и анионным ПАВ лаурилсульфатом натрия, которая имеет в присутствии некоторых аминов высокую чувствительность. Установлено образование при $\quad \mathrm{pH} \quad 6.2-8.5$ многокомпонентного комплекса с молярным коэффициентом поглощения $1.0 \cdot 10^{5}$ и соотношением Sc $:$ ПАВ $=1: 2$ [11].

Преимуществами полиэлектролитов перед ПАВ в качестве модификаторов реакции является низкая токсичность и стоимость. Поэтому целью работы было оценить возможность модификации наиболее широко используемых реагентов на $\mathrm{Sc}^{3+}$, а именно XAS и ЭХЦ, катионными полиакриламидами (КПАА) марки FO.

\section{Экспериментальная часть}

В работе использованы органические реагенты хромазурол $\mathrm{S}$ и эриоромцианин $\mathrm{R}$ фирмы "Fluka"; катионный флокулянт сополимер акриламида и метилхлорида ADAM фирмы "SNF Floerger" с плотностью заряда от 5 до 95\% и молекулярными массами $4 \cdot 10^{6}$ и $8 \cdot 10^{6}$ г/моль (рис. 1 ).

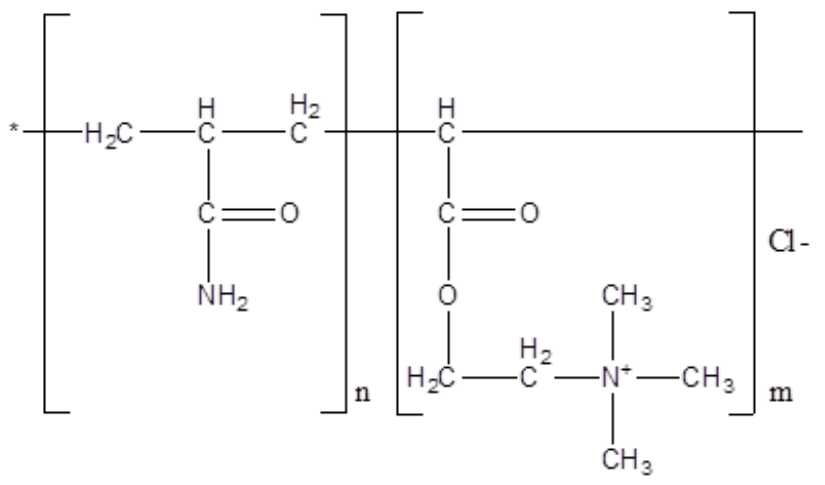

\begin{tabular}{|c|c|}
\hline Floculant & Charge density, \% \\
\hline FO4400 & 30 \\
\hline FO4650 & 55 \\
\hline FO4700 & 70 \\
\hline FO4990SH & 95 \\
\hline $\mathrm{M}_{\mathrm{r}}=4 \cdot 10^{6} \mathrm{~g} / \mathrm{mol}$ \\
$\mathrm{M}_{\mathrm{r}}(\mathrm{FO} 4990 \mathrm{SH})=8 \cdot 10^{6} \mathrm{~g} / \mathrm{mol}$ \\
\hline
\end{tabular}

Fig. 1. Structural formula, charge density and molecular weight of CPAA brand FO.

Рис. 1. Структурная формула, плотность заряда и молекулярная масса КПАА марки FO.

Растворы красителей XAS и ЭХЦ, раствор КПАА $(0.1$ г/л) готовили растворением точных навесок в бидистиллированной воде. Раствор $\mathrm{Sc}^{3+}\left(1 \cdot 10^{-3}\right.$ моль/л) готовили из стандартного образца СЗП 55-2013. Рабочие растворы готовили разбавлением исходных непосредственно перед экспериментом. Все реактивы, использованные в работе, были марки не ниже «х.ч.». Необходимые значения $\mathrm{pH}$ устанавливали раствором щелочи $(\mathrm{NaOH})$ или минеральной кислоты $\left(\mathrm{HNO}_{3}\right)$ и контролировали на универсальном иономере ЭВ - 74 со стеклянным электродом ЭСЛ - 6307 и хлоридсеребряным электродом сравнения. Оптическую плотность измеряли на спектрофотометре SPECORDM-40 и CФ-46 в кювете с толщиной слоя 2 см.

\section{Результаты и их обсуждение}

Максимумы светопоглощения комплексов Sc-XAS и Sc-ЭХЦ находятся при 540 и 535 нм, соответственно [10]. Введение КПАА позволяет получить тройные металлополимерные комплексы (ТМПК) $\mathrm{XAS}-\mathrm{FO}-\mathrm{Sc} \quad\left(\lambda_{\max }=620\right.$ нм $) \quad$ и $\quad$ ЭХЦ - FO - Sc $\left(\lambda_{\max }=590\right.$ нм). По спектрам на рис. 2 видно смещение максимума светопоглощения в присутствии КПАА (на 70 нм и 50 нм для XAS и ЭХЦ, соответственно) и увеличение оптической плотности (до $40 \%$ и $20 \%$ для XAS и ЭХЦ, соответственно).

Экспериментально установлено, что комплекс скандия с XAS и ЭХЦ образуется в области $\mathrm{pH}$ 4-7, максимальный выход комплекса соответствует $\mathrm{pH} 5$. Введение модификатора практически не влияет на интервал $\mathrm{pH}$ существования комплекса. Кривые зависимости оптической плотности растворов комплексов скандия с XAS от $\mathrm{pH}$ среды в отсутствии и присутствии КПАА имеют максимум при рН 5 (рис. За). При дальнейшем увеличении $\mathrm{pH}$, вплоть до $\mathrm{pH} 7$, оптическая плотность уменьшается на 1016\% (в присутствии FO с плотностью заряда $30-70 \%$ ) и на $40 \%$ (при добавлении FO c плотностью заряда 95\%), поэтому для дальнейших исследований выбрали рН 5. Зависимости оптической плотности растворов комплексов Sc - ЭХЦ от $\mathrm{pH}$ (рис. $3 \mathrm{~b}$ ) выходят на плато в интервале $\mathrm{pH} 5-7$ (в присутствии среднезаряженных образцов FO). Лишь при добавлении FO с плотностью заряда 95\% наблюдается незначительное уменьшение оптической плотности (рис. $3 \mathrm{~b}$, кр. 5). Оптимальным для комплексообразования $\mathrm{Sc}^{3+}$ с ЭХЦ выбрали значение pH 5.5 (рис. 3). 

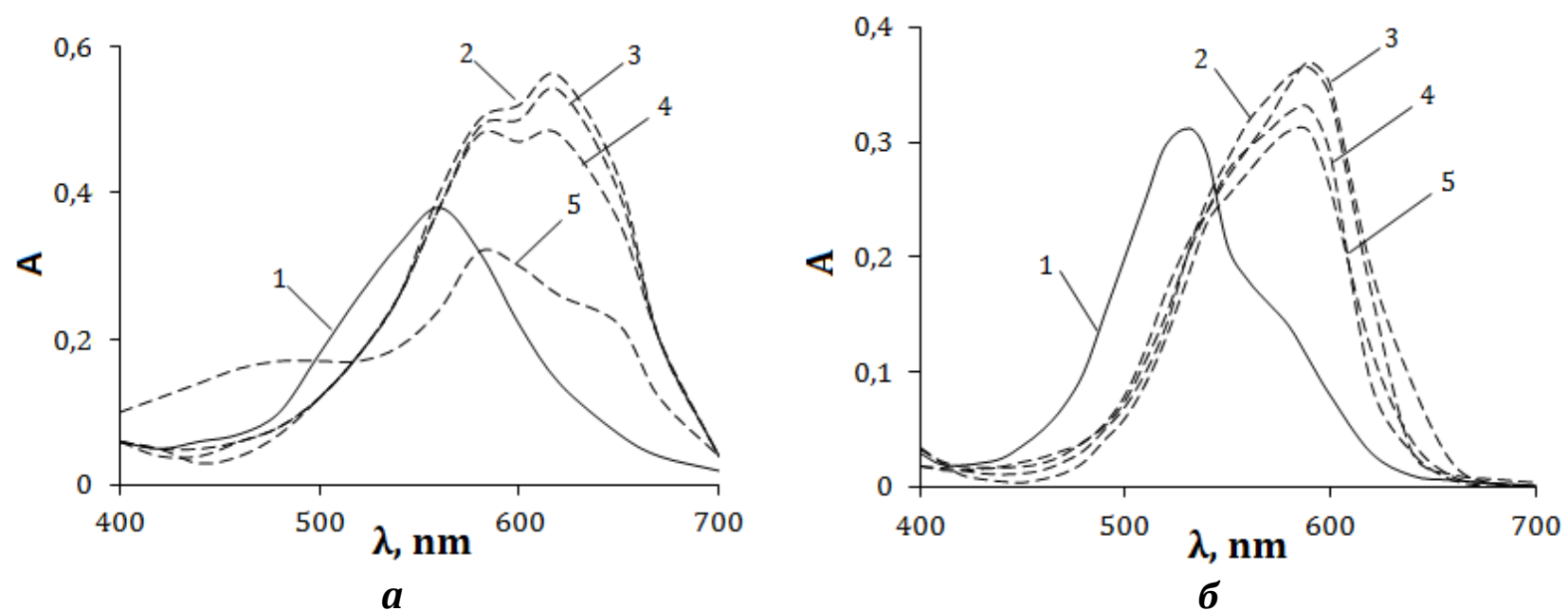

Fig. 2. Absorption spectra of scandium complexes with XAS (a) and ECC (b) in the absence (1) and the presence $\left(2\right.$ - 5) of CPAA. The charge density of CPAA,\%: 30 (2), 55 (3), 70 (4), 95 (5). $\mathrm{C}_{\mathrm{ECC}}=2 \cdot 10^{-5} \mathrm{~mol} / \mathrm{L}, \mathrm{CXAS}_{\mathrm{X}}=1.5 \cdot 10^{-5} \mathrm{~mol} / \mathrm{L}$, $\mathrm{C}_{\mathrm{FO}}=20 \mathrm{mg} / \mathrm{L}, \mathrm{C}_{\mathrm{Sc}}=2 \cdot 10^{-5} \mathrm{~mol} / \mathrm{L} ; \ell=20 \mathrm{~mm}, \mathrm{pH}=5.5(a), 5.0$ (b).

Рис. 2. Спектры поглощения комплексов скандия с XAS (a) и ЭХЦ (б) в отсутствии (1) и присутствии (2 - 5)

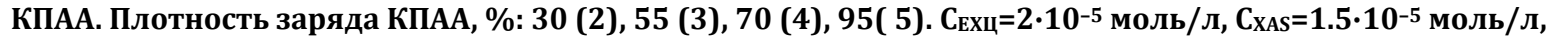
$\mathrm{C}_{\mathrm{FO}}=20$ мг $/$ Л, $\mathrm{Csc}_{\mathrm{sc}}=2 \cdot 10^{-5}$ моль/л; $\ell=2$ см, $\mathrm{pH}=5.5(a), 5.0$ (б).

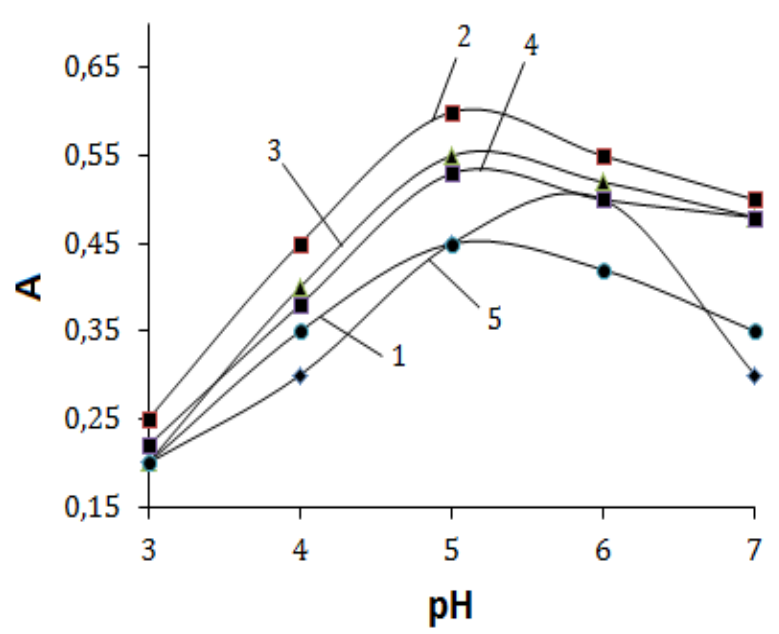

$a$

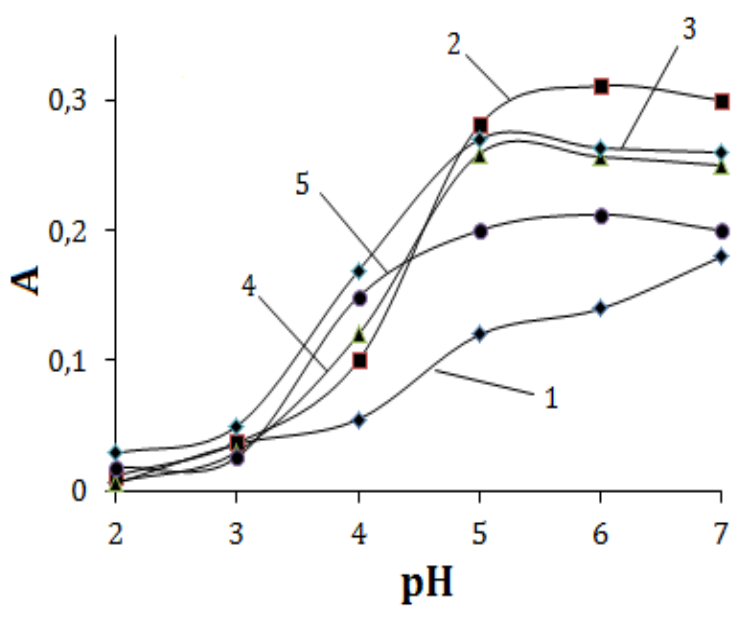

b

Fig. 3. Dependence of the optical density of scandium complexes with XAS (a) and ECC (b) in the absence (1) and the presence (2 - 5) of CPAA on the pH of the medium. The charge density of CPAA,\%: 30 (2), 55 (3), 70 (4), 95 (5).

$\mathrm{C}_{\mathrm{ECC}}=2 \cdot 10^{-5} \mathrm{~mol} / \mathrm{L}, \mathrm{C}_{\mathrm{XAS}}=1.5 \cdot 10^{-5} \mathrm{~mol} / \mathrm{L}, \mathrm{C}_{\mathrm{FO}}=20 \mathrm{mg} / \mathrm{L}, \mathrm{C}_{\mathrm{Sc}}=2 \cdot 10^{-5} \mathrm{~mol} / \mathrm{L} ; \ell=20 \mathrm{~mm}, \lambda=620 \mathrm{~nm}$ (a), $590 \mathrm{~nm}$ (b).

Рис. 3. Зависимость оптической плотности комплексов скандия с XAS (a) и ЭХЦ (b) в отсутствии (1) и присутствии (2 - 5) КПАА от рН среды. Плотность заряда КПАА, \%: 30 (2), 55 (3), 70 (4), 95(5).

$\mathrm{C}_{\text {ЕХц}}=2 \cdot 10^{-5}$ моль/л, СХАS=1.5 $10^{-5}$ моль/л, $\mathrm{C}_{F 0}=20$ мг/л, $\mathrm{C}_{S c}=2 \cdot 10^{-5}$ моль/л; $\ell=2 \mathrm{cM}, \lambda=620$ нм (a), 590 нм (b).

Изучено влияние концентрации полиакриламидов марки FO на аналитический сигнал системы краситель$\mathrm{FO}-\mathrm{Sc}$ (алгоритм приготовления ТМПК соответствует порядку записи компонентов тройной системы). Оптическая плотность растворов системы XAS-FO-Sc линейно возрастает в интервале концентраций $(0.5-15) \cdot 10^{-6}$ осново-моль/л (в пересчете на заряженные звенья). При концентрации модификатора более $15 \cdot 10^{-6}$ осново-моль/л, оптическая плотность практически постоянна. Соотношение компонентов FO : ЭХЦ соответствует $1: 2$, а для FO : XAS составляет $1: 1$. Составы комплексов Sc - XAS и Sc-ЭХЦ при $\mathrm{C}_{\mathrm{FO}}=8$ мкг/л не меняются независимо от плотности заряда полимерамодификатора, и равны $1: 4$ и $1: 6$, соответственно.

Для систем ЭХЦ - FO 4650 - Sc и XAS FO 4400 - Sc построены тройные диаграммы соотношения компонентов, которые отражают зависимость оптической плотности системы от концентрации трех компонентов 
(рис. 4). Замыкание изохром на диаграммах свидетельствует об образовании таких ТМПК, как ЭХЦ : FO 4650 : Sc состава $6: 3: 1$ (рис. 4a) и для косплекса XAS : FO $4400: \mathrm{Sc}$ составов $1: 1: 1$ и $1: 3: 1$ (рис. 4b). Преимуществом

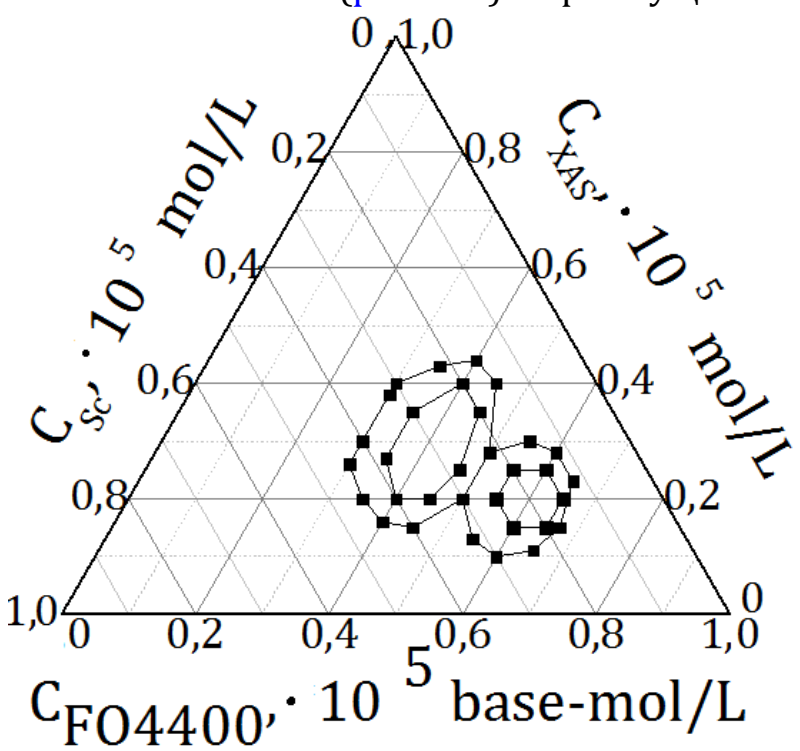

a системы ЭХЦ - FO 4700 - Sc перед XAS FO $4700-\mathrm{Sc}$ является образование одного комплекса, который выбран нами в качестве аналитической формы для определения скандия.

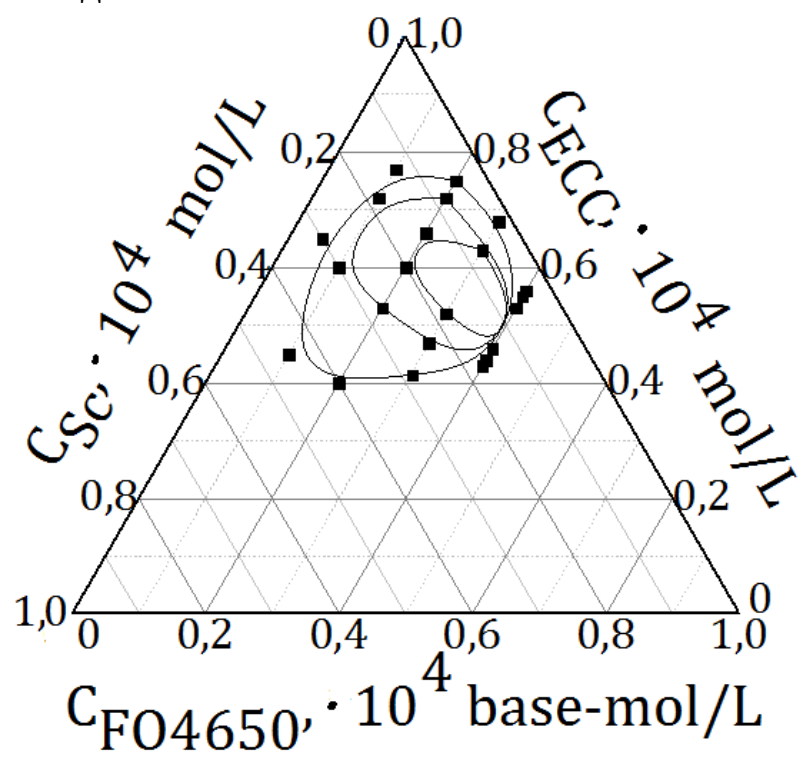

$\boldsymbol{b}$

Fig. 4. The ternary diagram of the components ratio in the systems XAS - FO $4400-\mathrm{Sc}^{3+}(a)$ and ECC FO $4650-\mathrm{Sc}^{3+}$ (b). (pH 5.0, $\left.\ell=20 \mathrm{~mm}, \lambda=590 \mathrm{~nm}(a), 620 \mathrm{~nm}(b)\right)$.

Рис. 4. Треугольник соотношения компонентов в системе ЕXЦ - FO 4650 - $\mathrm{Sc}^{3+}$ (a) и XAS - FO 4400 - $\mathrm{Sc}^{3+}$ (б). (рН 5.0; $\ell=2$ см; $\lambda=590$ нм (a), 620 нм (б)).

Оптическая плотность линейно зависит от концентрации $\mathrm{Sc}^{3+}$ в интервале 4.5 200 мкг/л при определении с модифицированным реагентом XAS-FO и 3.6 - 90 мкг/л при определении с ЭХЦ - FO. Для дальнейшего использования нами выбрана система ЭХЦ - FO 4700 - Sc как более чувствительная (табл. 1).

В табл. 2 приведены характеристики градуировочного графика для определения $\mathrm{Sc}^{3+}$ аналитической системой ЕХЦ - FO 4700 $\mathrm{Sc}^{3+}$. Предел обнаружения рассчитывали по формуле: $3 \mathrm{~S}_{\mathrm{a}} / \operatorname{tg} \alpha$; предел определения по формуле: $10 \mathrm{~S}_{\mathrm{a}} / \operatorname{tg} \alpha$, где $\mathrm{S}_{\mathrm{a}}$ - стандартное отклонение холостого опыта, tg $\alpha$ - угол наклона градуировочного графика.

Table 1

Molar absorbtivity of Sc complexes with ECC and XAS at modification by $\mathrm{FO}$ with different charge density

Таблица 1

Молярные коэффициенты светопоглощения комплексов Sc с ЭХЦ и XAS при модификации FO с разной плотностью заряда

\begin{tabular}{|c|c|c|c|c|}
\hline \multirow[b]{2}{*}{ Reagent } & \multicolumn{4}{|c|}{ Molar absorbtivity, $\mathrm{mol}^{-1} \cdot \mathrm{L} \cdot \mathrm{cm}^{-1}$} \\
\hline & F04400 & F04650 & F04700 & F04990SH \\
\hline XAS & $5.0 \cdot 10^{4}$ & $7.0 \cdot 10^{4}$ & $5.0 \cdot 10^{4}$ & $5.1 \cdot 10^{4}$ \\
\hline ECC & $6.3 \cdot 10^{4}$ & $5.3 \cdot 10^{4}$ & $7.2 \cdot 10^{4}$ & $4.2 \cdot 10^{4}$ \\
\hline
\end{tabular}

Table 2

Characteristics of the calibration graph for the determination of $\mathrm{Sc}^{3+}$ in the form $\mathrm{EXC}-\mathrm{FO} 4700$ - $\mathrm{Sc}^{3+}$

Характеристики градуировочного графика для определения $\mathrm{Sc}^{3+}$ в виде аналитической системы EXЦ - FO 4700 - $\mathrm{Sc}^{3+}$

\begin{tabular}{ccccc}
\hline $\begin{array}{c}\text { The calibration graph } \\
\text { equation }(\mathrm{C}, \mu \mathrm{g} / \mathrm{L})\end{array}$ & $\mathrm{R}^{2}$ & $\begin{array}{c}\text { Linearity interval, } \\
\mu \mathrm{g} / \mathrm{L}\end{array}$ & $\begin{array}{c}\text { Limit of } \\
\text { determination, } \mu \mathrm{g} / \mathrm{L}\end{array}$ & $\begin{array}{c}\text { Minimum Detectable } \\
\text { Value, } \mu \mathrm{g} / \mathrm{L}\end{array}$ \\
\hline $\mathrm{A}=0.069+3.099 \cdot \mathrm{C}$ & 0.9914 & $9.0-90.0$ & 5.5 & 15.5 \\
\hline
\end{tabular}

Изучение мешающего влияния посторонних ионов на определение $\mathrm{Sc}^{3+}$ в виде ЭХЦ(XAS) - FO 4700 - Sc

Хромазурол $\mathrm{S}$ и эриохромцианин $\mathrm{R}$ при pH 5 образуют комплекс с алюминием, который является основным компонентом сплава 01570. Поэтому нами изучено влияние КПАA марки FO на спектры комплекса алюминия с XAS и ЭХЦ (рис. 5) 


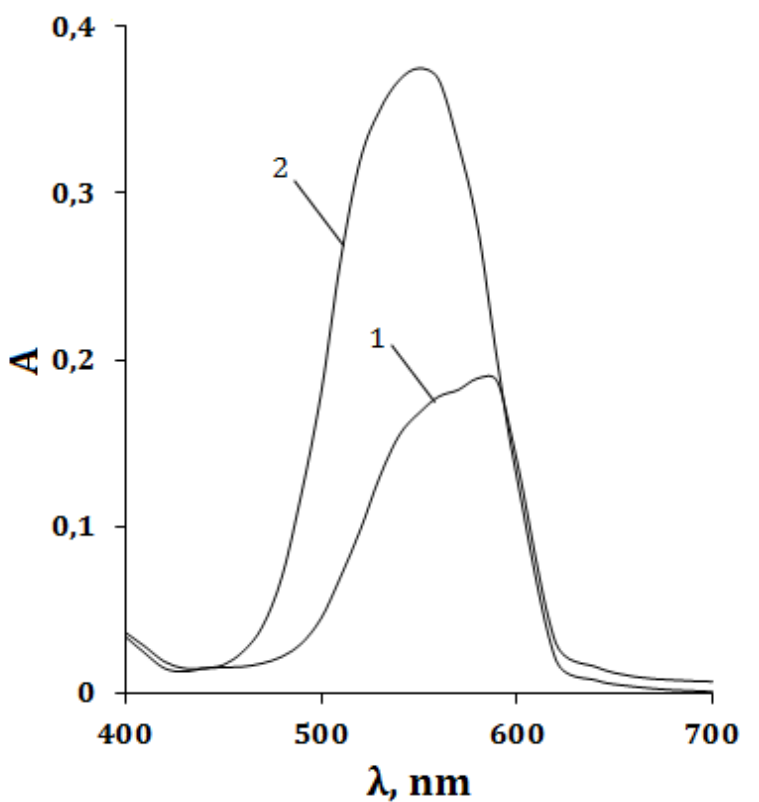

Fig. 5. Absorption spectra of the system EXC FO $4700-\mathrm{Sc}^{3+}$ (1) and ECC - FO $4700-\mathrm{Sc}^{3+}-\mathrm{Al}^{3+}$ (2). $\mathrm{C}_{\mathrm{ECC}}=2 \cdot 10^{-5} \mathrm{~mol} / \mathrm{L}, \mathrm{C}_{\mathrm{FO}}=20 \mathrm{mg} / \mathrm{L}, \mathrm{CSC}_{\mathrm{SC}}=2 \cdot 10^{-5} \mathrm{~mol} / \mathrm{L}$; $\mathrm{C}_{\mathrm{Al}}=2 \cdot 10^{-5} \mathrm{~mol} / \mathrm{L}, \mathrm{pH} 5.0, \ell=20 \mathrm{~mm}$

Рис. 5. Спектры поглощения системы ЕХЦ FO $4700-\mathrm{Sc}^{3+}$ (1) и EXЦ - FO $4700-\mathrm{Sc}^{3+}-\mathrm{Al}^{3+}$ (2). СЕХЦ $=2 \cdot 10^{-5}$ моль $/$ л, $\mathrm{C}_{\mathrm{FO}}=20$ мг $/$ л, $\mathrm{C}_{\mathrm{Sc}}=2 \cdot 10^{-5}$ моль $/$ л; $\mathrm{C}_{\mathrm{Al}}=$ $2 \cdot 10^{-5}$ моль/л, рН 5.0, $\ell=2$ см.

Проведением модификации ЭХЦ или XAS вдением FO 4700 перед определением $\mathrm{Sc}^{3+}$ не удалось устранить мешающее влияние ионов алюминия, которое проявляется даже при двукратном избытке $\mathrm{Al}^{3+}$. Скандий рекомендуют отделять от алюминия осаждением с $\mathrm{NaOH}$ [10]. При осаждении раствором $\mathrm{NaOH}$ сопутствующие скандию редкоземельные элементы, $\mathrm{Mn}$ и $\mathrm{Fe}$ осаждаются; а щелочные и щелочноземельные металлы, $\mathrm{Be}, \mathrm{Al}, \mathrm{W}$ и Мо остаются в растворе. Мешающее влияние других компонентов сплава 01570 на оптическую плотность системы ЕХЦ - FO $4700-\mathrm{Sc}^{3+}$ представлено в табл. 3.

Осаджение Sc проводили из теплого раствора при помощи 1 моль/л раствора $\mathrm{NaOH}$. Фильтровали через стеклянный фильтр, полученный осадок растворяли в разбавленной азотной кислоте. Для спектрофотометрического определения скандия в колбе на 25 мл готовили раствор, содержащий 5 мл растворенного осадка, 0.5 мл $1 \cdot 10^{-3} \mathrm{M}$ раствора ЭХЦ и 1 мл 0.1 г/л раствора FO 4700. Параллельно, в колбе на 25 мл готовили стандартный раствор, содержащий 0.5 мл $1 \cdot 10^{-3}$ М раствора ЕХЦ, 1 мл 0.1 г/л раствора FO 4700 (концентрация скандия в стандартном растворе соответствовала его концентрации в 5 мл растворенного осадка). Устанавливали рН 5.5 добавлением растворов $\mathrm{HNO}_{3}\left(\begin{array}{ll}0.1 & \mathrm{M}\end{array}\right)$, контролируя значение $\mathrm{pH}$ на иономере. Объем доводили до метки бидистиллированной водой и измеряли оптическую плотность при 590 нм в кюветах с $\ell=5$ см (табл. 5).

Influence of the components of alloy 01570 on the absorbance of the system ECC - FO $4700-\mathrm{Sc}^{3+}$

Таблица 3

Влияние компонентов сплава 01570 на оптическую плотность системы ЕХЦ - FO 4700 - Sc ${ }^{3+}$

\begin{tabular}{lll}
\hline $\begin{array}{l}\text { The interfering } \\
\text { component }\end{array}$ & $\begin{array}{l}\text { Concentration, } \mathrm{M} \\
\text { (ratio Sc: } \mathrm{Me})\end{array}$ & $\Delta \mathrm{A}, \%$ \\
\hline $\mathrm{Al}^{3+}$ & $1 \cdot 10^{-2}(1: 500)$ & 200 \\
$\mathrm{Fe}^{3+}$ & $4 \cdot 10^{-5}(1: 2)$ & 4.2 \\
$\mathrm{Cu}^{2+}$ & $2 \cdot 10^{-5}(1: 1)$ & 4.1 \\
$\mathrm{Zn}^{2+}$ & $2 \cdot 10^{-5}(1: 1)$ & 4.1 \\
$\mathrm{Mg}^{2+}$ & $8 \cdot 10^{-4}(1: 40)$ & 2.6 \\
\hline
\end{tabular}

Методика спектрофотометрического определения $\mathrm{Sc}^{3+}$ после предварительного осаждения из модельного раствора сплава 01570.

Модельный раствор сплава 01570 готовили согласно данным табл. 4.

Table 4

Data for the preparation of model solution of alloy 01570

Таблица 4

Данные для приготовления модельного раствора сплава 01570

\begin{tabular}{llll}
\hline Metal & $\mathrm{C}, \mathrm{mol} / \mathrm{L}$ & Metal & $\mathrm{C}, \mathrm{mol} / \mathrm{L}$ \\
\hline $\mathrm{Sc}$ & $5.0 \cdot 10^{-5}$ & $\mathrm{Zn}$ & $1.7 \cdot 10^{-5}$ \\
$\mathrm{Al}$ & $1.0 \cdot 10^{-2}$ & $\mathrm{Mg}$ & $7.5 \cdot 10^{-4}$ \\
$\mathrm{Fe}(\mathrm{II})$ & $4.0 \cdot 10^{-5}$ & $\mathrm{Mn}(\mathrm{II})$ & $1.0 \cdot 10^{-4}$ \\
$\mathrm{Cu}$ & $1.7 \cdot 10^{-5}$ & & \\
\hline
\end{tabular}

The optical density of solutions at $590 \mathrm{~nm}$

Table 5

Таблица 5

Значение оптической плотности растворов при 590 нм

\begin{tabular}{ll}
\hline $\begin{array}{l}\text { Analytical system } \\
\mathrm{ECC}-\mathrm{FO} 4700-\mathrm{Sc}^{3+}\end{array}$ & $\begin{array}{l}\text { Model alloy dissolved after } \\
\text { precipitation }\end{array}$ \\
\hline 0.31 & 0.30 \\
\hline
\end{tabular}

Как видно из табл. 5, предварительное осаждение ионов $\mathrm{Sc}^{3+}$ позволяет устранить мешающее влияние $\mathrm{Al}^{3+}$ и проводить дальнейшее определение $\mathrm{Sc}^{3+}$ в виде системы ЕХЦ - FO $4700-\mathrm{Sc}^{3+}$. На основании экспериментальных данных разработана методика спектрофотометрического определения $\mathrm{Sc}^{3+}{ }^{+}$сплаве 01570. 
Методика спектрофотометрического определения $\mathrm{Sc}^{3+}$ в сплаве 01570. Для построения градуировочного графика в колбы на 25 мл вносили 0.5 мл 1·10-3 моль/л раствора ЕХЦ, 1 мл 0.1 г/л раствора FO 4700, 0 - 1.2 мл (с шагом по 0.2 мл) раствора $\mathrm{Sc}^{3+}$ $\left(2 \cdot 10^{-4}\right.$ моль/л), устанавливали $\mathrm{pH} 5.5$ и измеряли оптическую плотность при длине волны 590 нм.

Для анализа брали навеску сплава 01570 (содержание скандия от 0.15 до $0.35 \%$ ) массой 0.2500 г, растворяли при нагревании в концентрированной $\mathrm{HNO}_{3}$. После полного растворения минерализат кипятили втечение 2 мин. Охлажденный минерализат переносили в мерную колбу емкостью 100 мл и доводили до метки бидистиллированной водой. Для анализа отбирали аликвоту минерализата объемом 1 мл и проводили осаждение и определение по описанной выше методике. Результаты определения $\mathrm{Sc}^{3+}$ проверены альтернативной методикой по ГОСТ 11739.25-90 [24]. Результаты определения представлены в табл. 6. Проверка по критерию Фишера $\left(\mathrm{F}_{\text {эксп }}<\mathrm{F}_{\text {табл }}\right)$ подтвердил отсутствие различий в воспроизводимостях результатов. Правильность предложенной и стандартной методики проверяли по критерию Стьюдента $\left(\mathrm{t}_{\text {эксп }}<\mathrm{t}_{\text {табл }}\right)$, который показал отсутствие систематической погрешности при определении $\mathrm{Sc}^{3+}$.

Table 6

Results of Scandium content determination in alloy 01570 by developed and alternative technique Таблица 6

Результаты определения содержания скандия в сплаве 01570 разработанной и альтернативной методикой

\begin{tabular}{lll}
\hline Technique & ECC - FO $4700-\mathrm{Sc}^{3+}$ & GOST 11739.25-90 \\
\hline Sc, $\%$ & 0.266 & 0.260 \\
Sr & 0.054 & 0.056 \\
F-test & & F $_{\text {experimental }}=1.8<\mathrm{F}_{0.95 ; 2 ; 2}=19$ \\
t-test & & texperimental $=0.6<\mathrm{t}_{0.95 ; 2 ; 2}=4.3$ \\
\hline
\end{tabular}

Note: $\mathrm{F}_{(\mathrm{P} ; \mathrm{f1} ; \mathrm{f2})}-$ Fisher's test; $\mathrm{t}_{(\mathrm{P} ; \mathrm{f} 1 ; \mathrm{f} 2)}-$ Student's t test.

\section{Выводы}

Изучено комплексообразование скандия с хромазуролом $\mathrm{S}$ и эриохромцианином $\mathrm{R}$ в присутствии КПАА марки FO с различной плотностью заряда. Среди ряда изученных КПАА марки FO подобран наиболее эффективный модификатор для XAS и ЭХЦ FO 4700. Соотношение, позволяющее получить максимальный аналитический сигнал, определено по тройной диаграмме и составляет ЕХЦ : FO : Sc = $6: 3: 1$. Система

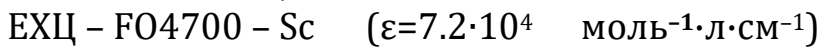
предложена как аналитическая форма для спектрофотометрического определения скандия в интервале концентраций $\mathrm{Sc}^{3+}$ 9 - 90 мкг/л. Методика апробирована при анализе образца скандийсодержащего сплава 01570, после предварительного отделения от мешающих компонентов осаждением с $\mathrm{NaOH}$. Предел определения $\mathrm{Sc}^{3+}$ по предложенной методике составляет 5.5 мкг/л. Правильность определения подтверждена стандартной методикой по ГОСТ 11739.25-90.

\section{Библиографические ссылки}

[1] Lim Y.P. Effect of heat treatment on gravity die-cast ScA356 aluminium alloy / Y. P. Lim W. H. Yeo A. Masita // Manufacturing Rev. - 2017, Vol.4, N 3. - P. 1-4.

[2] Effect of Sc addition on the microstructure and wear properties of A356 alloy and A356-TiB2 in situ composite / S. L. Pramod, A. K. Prasada Rao, S. R. Bakshi // Materials and Design. - 2015. Vol. 78. P. 85-94.

[3] Effect of Sc addition on microstructure and mechanical properties of 1460 alloy / J. Ma, D. Yan, L. Rong, Y. Li // Progress in Natural Science: Materials International. 2014. - Vol. 24. - P. 13-18.

[4] Повышение механических свойств при изменении структуры способом наномодификации алюминиевых сплавов / Н. Е. Калинина, Е. А. Джур, 3. В. Вилищук [и др.] // Вісник Дніпропетровського університету. Серія: Ракетно-космічна техніка. 2013. - T. 21, N 4. - С. 29-33.

[5] Изменение структуры и свойств литейных алюминиевых сплавов при дисперсном модифицировании / Н. Е. Калинина, Е. А. Мусина, В. Т. Калинин [и др.] // Металознавство та термічна обробка металів. - 2014 - Т. 65, N 2. - С. 51-56.

[6] Спектрофотометрическое определение скандия (III) в вулканогенной породе с помощью бис-(2,3,4триоксифенилазо) бензидина и алифатических аминов / Р. А. Алиева, С. Р. Гаджиева, Т. И. Алиева и др. // Молодой ученый. - 2012. - Т. 38, N 3. - С. 105108.

[7] Гаджиева С. Р. Новая методика фотометрического определения скандия (III) в прикаспийской светлокаштановой почве с бис- $(2,3,4-$ тригидроксифенилазо) бензидином в присутсвии диантипирилметана и его гомологов / С. Р. Гаджиева, Т. И. Алиева, Ф. М. Чырагов // Химия и химическая технология. - 2008. - Т. 51, N 10. C. 48-51.

[8] Егорова К.А. Атомно-абсорбционное определение скандия в растворах сложного солевого состава / К. А. Егорова, Ю. В. Соколова // Заводская лаборатория. Диагностика материалов. - 2001. T. 67, N 7. - C. 21-22.

[9] Жерноклеева К. В. Анализ чистых скандия, иттрия и их оксидов методами атомно-эмисионной спектрометрии с индуктвно-связаной плазмой и масс-спектрометрии с индуктивно-связаннной плазмой / К. В. Жерноклеева, В. Б. Барановская // 
Заводская лаборатория. Диагностика материалов. 2010. - T. 76, N 11. - С. 20-26.

[10] Комиссарова Л. Н. Аналитическая химия скандия / Л. Н. Комиссарова. - М.: Эдитроиал УРСС, 2001. $512 \mathrm{c}$.

[11] Sarsam L. A. Spectrophotometric determination of scandium (III) with eriochrome cyanine $\mathrm{R}$ and cetylpyridinium chloride - application to waters and synthetic alloys / L. A. Sarsam, W. A. Bashir // J. Raf. Sci. - 2009 - Vol. 20, N 3. - P. 48-65.

[12] Park Chan-il Spectrophotometric determination of scandium(III) in monazite after separation using Amberlite IRC 718 chelating resin / Chan-il Park, KiWon Cha // Bull. Korean Chem. Soc. - 1999. -Vol. 20, N 12. -P. 1409-1412.

[13] Изучение спектрофотометрического определения скандия в смеси редкоземельных элементов с применением хромазурола $\mathrm{S}$ в присутствии бромида цетилпиридиния / Z. Huanzhen, W. Zhi, M.Fengzhi [et al.] // Phys. Test. and Chem. Anal. B: Chem. Anal. - 1995. - Vol. 31, N 5. - P. 276-278.

[14] Влияние водорастворимого полисульфонилпиперидинилметиленгидроксида на взаимодействие скандия (III) с хромазуролом S / Т. С. Чмиленко, 3. Г. Ольхова, Л. П. Сидорова и др. // Вопросы химии и химтехнологии. - 2007, N 4. C. 23-27.

[15] Karve M. Solvent extraction and determination of scandium (III) with Cyanex 272 as an extractant / M. Karve, B. Vaidya // Indian J. Chem. - 2006. - Vol. 54(A). - P. 2658-2660.

[16] Курбатова Л. Д. Спектрофотометрическое определение скандия с хлорцианформазаном / Л. Д. Курбатова, Д. И. Курбатов // Заводская лаборатория. Диагностика материалов. - 2006. T. 72, N 9. - C. 18-20.

[17] Bieke 0. Recovery of scandium (III) from aqueous solutions by solvent extraction with the functionalized ionic liquid betainium bis(trifluoromethylsulfonyl)imide / O. Bieke, K. Binnemans // Ind. Eng. Chem. Res. - 2015. - Vol. 54. - P. 1887-1898

[18] Симонова Т. Н. Экстракция роданидных комплексов скандия в двухфазных водных системах / Т.Н.Симонова, А. Н. Федотов, А. С. Белодед // Укр. хим. журн. - 2008. - Т. 74, N 8. С. $113-117$.

[19] Симонова Т. Н. Экстракционное извлечение и определение разнозарядных ацидокомплексов скандия (III) и церия(IV) в двухфазных водных системах / Т. Н. Симонова, А. Н. Федотов // Вісник Харківського нац. ун-ту. - 2007. - Вип. 15 (38), N 770. - C. $132-136$

[20] Симонова Т. Н. Экстракция и спектрофотометрическое определение скандия в двуфазной водной системе полиэтиленгликольнитрит натрия-вода / Т. Н. Симонова, А. Н. Федотов // Методы и объекты химического анализа. - 2007. - T. 2, N 1, C. 51-55.

[21] Чмиленко Т. С. Аналитическая химия полиэлектролитов и их применение в анализе / Т. С. Чмиленко, Ф. А. Чмиленко. - Д.: Изд-во ДНУ, 2012. - $224 \mathrm{c}$.

[22] Sydorova L. Polyelectrolyte effect on spectrophotometric determination and interaction of scandium (III) with eriochromcyanine / L. Sydorova, A. Chernyavska, T. Chmylenko, F. Chmylenko //
Eastern European Journal of Enterprise Technologies. 2017. - Vol. 4, N 6(88). - P.53-58.

[23] Ассоциаты полисульфонилпиперидинилметиленгидроксида с сульфофталеинами и их химико-аналитические свойства / Ф. А. Чмиленко, Ю. М. Пискун, Т. С. Чмиленко и др. // Журн. аналит. химии. - 1997. - Т. 52, N 7. - С. 703-706.

[24] ГОСТ 11739.25-90 Сплавы алюминиевые литейные и деформируемые. Методы определения скандия. Введ. 1991-30-06. - М.: ИПК Изд-во стандартов, 1990. -7 c.

\section{References}

[1] Lim, Y.P., Yeo, W.H., Masita, A. (2017) Effect of heat treatment on gravity die-cast Sc-A356 aluminium alloy Manufacturing Rev. $4 \quad$ (3) 1 - 4. https://doi.org/10.1051/mfreview/2017003

[2] Pramod, S.L. Prasada Rao, A.K. Bakshi, S.R. (2015) Effect of Sc addition on the microstructure and wear properties of A356 alloy and A356-TiB2 in situ composite, Materials and Design (78) 85-94. https://doi.org/10.1016/j.matdes.2015.04.026

[3] J. Ma, D. Yan, L. Rong, Y. Li, (2014) Effect of Sc addition on microstructure and mechanical properties of 1460 alloy, Progress in Natural Science: Materials International (24) 13-18. https://doi.org/10.1016/i.pnsc.2014.01.003

[4] Kalinina, N.E., Dzhur, E.A., Vilishchuk, Z.V. Nosova, T.V., Agapov,V.A. (2013) [Increase of mechanical properties when the structure is changed by the method of nanomodification of aluminum alloys]. Visnyk Dnipropetrovs'koho universytetu. Seriya: Raketnokosmichna tekhnika - Bulletin of Dnipropetrovsk University. Series: Rocket and Space Technology. 21 (4), 29-33 (in Russian). http://www.dnu.dp.ua/docs/visnik/ftf/program 56b2 4a85067c8.pdf

[5] Kalinina, N. E., Musina, E. A, Kalinin, V. T., Vilishchuk, Z. V., Bekesh, E. C (2014) [Changes in the structure and properties of cast aluminum alloys with a dispersed modification]. Metaloznavstvo ta termichna obrobka metaliv - Metallurgy and thermal treatment of metals. 65 (2), 51-56 (in Russian).

[6] Alieva, R. A., Gadzhiyeva, S. R, Aliyev, T. I., Chyragov, F. M., Rafiyeva. G. L., Abdullayev, R. A. (2012) [Spectrophotometric determination of scandium (III) in volcanogenic breed with the use of bis (2,3,4-trioxyphenylazo) benzidine and aliphatic amines]. Molodoy uchenyy - Young Scientist. 38 (3), 105-108 (in Russian). https://moluch.ru/archive/38/4332

[7] Gadzhiyeva, S. R., Aliyeva, T. I., Chyragov, F. M. (2008) [A new technique for the photometric determination of scandium (III) in the Caspian light chestnut soil with bis (2,3,4-trihydroxyphenylazo) benzidine in the presence of diantipyrylmethane and its homologues]. Khimiya i khimicheskaya tekhnologiya - Chemistry and chemical technology, 51 (10), 48-51 (in Russian).

[8] Yegorova, K. A., Sokolova, Yu. V. (2001) [Atomicabsorption determination of scandium in solutions of complex salt composition]. Zavodskaya laboratoriya. Diagnostika materialov - Factory laboratory. Material diagnostics, 67(7), 21-22 (in Russian)

[9] Zhernokleyeva, K. V. Baranovskaya, V. B. (2010) [Analysis of pure scandium, yttrium and their oxides by methods of atomic-emission spectrometry with an 
inductively-coupled plasma and inductively-coupled plasma mass spectrometry]. Zavodskaya laboratoriya. Diagnostika materialov - Factory laboratory. Material diagnostics, 76 (11), 20-26 (in Russian)

[10] Komissarova, L. N. (2001). [Analytical chemistry of scandium]. Moskow, Russian Federation: Editroial URSS (in Russian).

[11] Sarsam, L. A., Bashir, W. A. (2009) Spectrophotometric determination of scandium (III) with eriochrome cyanine $\mathrm{R}$ and cetylpyridinium chloride - application to waters and synthetic alloys. J. Raf. Sci., 20 (3), 48-65. https://www.iasj.net/iasj?func=fulltext\&aId $=39972$

[12] Park, Chan-il, Cha, Ki-Won (1999). Spectrophotometric determination of scandium(III) in monazite after separation using Amberlite IRC 718 chelating resin. Bull. Korean Chem. Soc. 20 (12), 1409-1412. http://pdf.easechem.com/pdf/32/d985ffcb-6b8b40f6-9d6e-775c7fc343b0.pdf

[13] Huanzhen, Z., Zhi, W., Fengzhi, M., Jinghu, Z., Honggao, L. (1995) [The study of spectrophotometric determination of scandium in a mixture of rare-earth elements with using chromazurol $\mathrm{S}$ in the presence of cetylpyridinium bromide] Phys. Test. and Chem. Anal. B: Chem. Anal. 31 (5), 276-278.

[14] Chmilenko, T. S., Ol'khova, Z. G., Sidorova, L. P., Chmilenko, F. A. (2007) [Effect of water-soluble polysulfonylpiperidinylmethylene hydroxide on the interaction of scandium (III) with chromazurol S]. Voprosy khimii i khimicheskoi technologii - Issues of Chemistry and Chemical Technology. 4, 23-27 (in Russian).

[15] Karve, M. Vaidya, B. (2006) Solvent extraction and determination of scandium (III) with Cyanex 272 as an extractant. Indian J. Chem. 54 (A), 2658-2660. http://nopr.niscair.res.in/bitstream/123456789/1800 9/1/IJCA\%2045A(12)\%202658-2660.pdf

[16] Kurbatova, L. D. Kurbatov, D. I. (2006) [Spectrophotometric determination of scandium with chlorcyanformazane]. Zavodskaya laboratoriya. Diagnostika materialov - Factory laboratory. Material diagnostics, 72(9), 18-20, (in Russian). http://www.zldm.ru/upload/iblock/8db/2006-9-1820.pdf

[17] Onghena, Bieke, Binnemans, Koen (2015) Recovery of scandium (III) from aqueous solutions by solvent extraction with the functionalized ionic liquid betainium bis(trifluoromethylsulfonyl)imide. Ind. Eng. Chem. Res. 54, 1887-1898.
[18] Simonova, T. N., Fedotov, A. N., Beloded A. S. (2008) [Extraction of rhodanide scandium complexes in twophase aqueous systems]. Ukrainskiy khimicheskiy zhurnal - Ukrainian chemical journal, 74(8), 113-117 (in Russian). http://dspace.nbuv.gov.ua/bitstream/handle/123456 789/15906/09-Simonova.pdf

[19] Simonova, T. N., Fedotov, A. N. (2007) [Extraction and determination of multiply charged azido complexes of scandium (III) and cerium (IV) in two-phase aqueous systems]. Visnyk Kharkivs'koho natsional'noho universytetu - Bulletin of Kharkiv National University, 15(770), 132-136 (in Russian). http://chembull.univer.kharkov.ua/archiv/2007/17.p $\underline{\mathrm{df}}$

[20] Simonova, T. N., Fedotov, A. N. (2007) [Extraction and spectrophotometric determination of scandium in a two-phase aqueous sodium-polyethylene glycol-nitrite aqueous system] Metody Ob'ekty Khim. Anal. - Methods Objects Chem. Anal., 2(1), 51-55 (in Russian). http://chembull.univer.kharkov.ua/archiv/2007/17.p $\underline{\mathrm{df}}$

[21] Chmilenko, T. S., Chmilenko, F. A. (2012) [Analytical chemistry of polyelectrolytes and their application in analysis]. Dnipropetrovsk, Ukraine: Izd-vo DNU (in Russian)

[22] Sydorova, L., Chernyavska, A., Chmylenko, T., Chmylenko, F. (2017) [Polyelectrolyte effect on spectrophotometric determination and interaction of scandium (III) with eriochromcyanine] Eastern European Journal of Enterprise Technologies, 4(6-88), 53-58. $\quad$ https://doi.org/10.15587/17294061.2017.108371

[23] Chmilenko, F. A., Piskun, Yu. M., Chmilenko, T. S., Rysinskaya E. A. (1997) [Associates of polysulfonylpiperidinylmethylene hydroxide with sulfophthaleins and their chemical-analytical properties] Zhurnal analiticheskoy khimii - Journal of Analytical Chemistry, 52(7), 703-706 (in Russian).

[24] Federal Agency on Technical Regulating and Metrology (1990) [Aluminium casting and wrought alloys. Methods for determination of scandium](GOST 11739.25-90), Moskow, USSR: Izdatelstvo standartov (in Russian) https://uploaddd3a096b86c38e430c9a800274739224.hb.bizmrg.co m/iblock/268/2685b32c277d185ba6620de7f09c3a2f 119291.pdf 